\title{
Zr semanter
}

\section{Assessment of Modified Cerebral Blood Flow and Its Connectivity With Migraines Without Aura During Interictal Periods by Arterial Spin Labeling}

\section{Di Zhang}

Nanjing First Hospital https://orcid.org/0000-0003-4153-9283

\section{Xiaobin Huang}

Nanjing Medical University affiliated Nanjing Hospital: Nanjing First Hospital

\section{Cunnan Mao}

Nanjing Medical University affiliated Nanjing Hospital: Nanjing First Hospital

\section{Yuchen Chen}

Nanjing Medical University affiliated Nanjing Hospital: Nanjing First Hospital

\section{Zhengfei Miao}

Nanjing Medical University affiliated Nanjing Hospital: Nanjing First Hospital

Chunmei Liu

Nanjing Medical University affiliated Nanjing Hospital: Nanjing First Hospital Chenjie Xu

Nanjing Medical University affiliated Nanjing Hospital: Nanjing First Hospital

\section{Xinying Wu}

Nanjing Medical University affiliated Nanjing Hospital: Nanjing First Hospital

Xindao Yin ( $\nabla$ y.163yy@163.com )

Nanjing Medical University affiliated Nanjing Hospital: Nanjing First Hospital

\section{Research article}

Keywords: Migraine without aura, arterial spin labeling MRI, perfusion imaging, regional cerebral blood flow

Posted Date: April 30th, 2021

DOI: https://doi.org/10.21203/rs.3.rs-470813/v1

License: (c) (i) This work is licensed under a Creative Commons Attribution 4.0 International License. Read Full License 
Version of Record: A version of this preprint was published at The Journal of Headache and Pain on July 14th, 2021. See the published version at https://doi.org/10.1186/s10194-021-01282-y. 


\section{Abstract}

Background: Migraine constitutes a global health burden, and its pathophysiology is not well-understood; research evaluating cerebral perfusion and altered blood flow between brain areas using non-invasive imaging techniques, such as arterial spin labeling, have been scarce. This study aimed to assess cerebral blood flow (CBF) and its connectivity of migraine.

Methods: This study enrolled 40 patients with episodic migraine without aura (MwoA), as well as 42 healthy patients as control $(\mathrm{HC})$. Two groups of normalized CBF and CBF connectivity were compared, and the relationship between $\mathrm{CBF}$ variation and clinical scale assessment was further evaluated.

Results: In comparison to $\mathrm{HC}$ subjects, MwoA patients exhibited higher $\mathrm{CBF}$ in the right middle frontal orbital gyrus (ORBmid.R) and the right middle frontal gyrus, while that in Vermis_6 declined. The increased CBF of ORBmid.R was positively correlated with both the Visual Light Sensitivity Questionnaire8 (VLSQ-8) and the monthly attack frequency score. In MwoA, significantly decreased CBF connectivity was detected between ORBmid.R and the left superior frontal gyrus, the right putamen, the right caudate, as well as the right angular gyrus. In addition, increased CBF connectivity was observed between the left calcarine cortex and ORBmid.R.

Conclusions: Our results indicate that migraine patients exhibit abnormalities in regional CBF and feature CBF connection defects at the resting state. The affected areas involve information perception, information integration, and emotional, pain and visual processing. Our findings might provide important clues for the pathophysiology of migraine.

\section{Background}

For the period 1990-2017, migraine, a prevalent primary headache disorder, was considered the second most frequent factor associated with disability-adjusted life years according to the Global Burden of Disease study [1]. Epidemiological studies estimate that migraine affects 15 to 25 percent of women and 6 to 8 percent of men worldwide [2]. The condition is characterized by recurrent throbbing headache attacks, often accompanied by nausea, vomiting, photophobia, phonophobia, or allodynia [1,2]. Despite its significance, the pathogenesis of migraine has not been fully established. Advanced structural and functional imaging studies have provided some clues for understanding the pathophysiology of migraine and migraine-related dysfunction. The trigeminal neurovascular hypothesis is a comprehensive hypothesis that has played a leading role in explaining the pathogenesis of migraine [3]. Triptan drugs are clinically effective anti-migraine drugs developed according to this hypothesis. Triptans only have a slight constriction effect on blood vessels under physiological conditions, but can make abnormally dilated blood vessels and meningeal blood vessels constrict significantly [4]. The visual cortex, the limbic system, as well as pain and cognitive networks are also involved in the process of migraine [5-10].

According to the trigeminal neurovascular theory, changes in cerebral blood flow might occur during migraine attacks. Thus far, some studies have reported on cerebral perfusion changes in migraine 
patients. These are mostly case reports and tend to concentrate on migraine patients with aura. Research on perfusion imaging include the widely applied techniques of positron emission tomography (PET), CT perfusion, single photon-emission computed tomography (SPECT), dynamic susceptibility contrast (DSC), magnetic resonance perfusion imaging and perfusion-weighted imaging (PWI) [11-13]. One of the non-invasive cerebral perfusion imaging techniques is arterial spin labeling (ASL), that can be utilized to quantitatively assess the degree of cerebral perfusion without having to apply a gadolinium-based contrast agent $[14,15]$. The results of ASL are consistent with PET and DSC, and ASL provides a brain CBF measurement with high reliability and repeatability $[16,17]$. Pseudo-continuous ASL (PCASL) employs a long series of radiofrequency pulses and gradient pulses without the need to modify the scanner hardware. This method adopts a fast spin echo sequence and helically fills the $\mathrm{K}$ space with higher resolution. It can be used for whole-brain scanning and has a high clinical application value [14, $15,18,19]$. Due to its hybrid nature, PCASL is the most commonly used sequence in modern ASL imaging $[18,19]$. Perfusion abnormalities have been generally described by qualitative assessment. Most studies confirmed the detection of cerebral hypoperfusion in the acute phase of aura followed by rebound hyperperfusion $[20,21]$ and hyperperfusion in the onset phase[22-24]. In contrast to the typical stroke pattern, the hypoperfusion of brain tissue in the aura phase is not distributed according to the vascular blood supply area, which supports the neurogenic rather than the vascular origin of migraine aura [20].

To date, few studies have employed ASL imaging to evaluate the interictal perfusion of migraine without aura (MwoA), and their results were not consistent. The presence of hypoperfusion in brain areas was confirmed by ASL MRI during MwoA attacks. In particular, this technique detected a reduction in CBF [25]. A recent study indicated increased $\mathrm{CBF}$ in the ipsilateral dorsolateral pontine during MwoA attack using PCASL [26]. The ASL MRI method was also used to compare cerebral perfusion during migraine without aura attack and a headache-free period, while no global or regional differences were found [27]. The differences in the above results may be attributed to the fact that they were case reports or included small cohorts, and featured a diversity of disease severity, accompanying disorders, scanning methods or time interval from onset to examination. Considering that the brain is in a continuous internal metabolic activity state at rest, ASL provides the opportunity to detect and monitor changes in tissue perfusion that might indicate brain dysfunction. This study aimed to detect the pattern of CBF at the interictal phase of episodic MwoA and establish the relationships between changes in CBF and clinical scale assessment. In addition, we also investigated whether the abnormal CBF brain regions of MwoA patients also show changes in CBF connections. It is assumed that the links between cerebral perfusion and changes in cerebral blood flow in MwoA patients are different compared to those in healthy controls (HC), and these changes can be associated with a certain assessment scale in the clinical practice.

\section{Methods}

\subsection{Subject selection criteria}

This study was conducted with the approval of the Ethics Committee of Nanjing First Hospital, Nanjing, China. All subjects signed the informed consent forms. A total of 55 patients were recruited from the pain 
clinic and the Neurology Department of Nanjing First Hospital between the period of May 2018 to April 2020. All of them had episodic MwoA, according to the International Classification of Headache Disorders (Third Edition, beta version; ICHD-3 beta), and were aged between 18-50 years. Based on matching for age, gender and number of years in education $44 \mathrm{HC}$ subjects were finally included in the study.

The following exclusion criteria were applied: (1) Poor image quality; (2) MRI contraindications, (3) neuropsychological disorders; (4) history of alcohol or substance abuse; (5) brain damage or other neurological diseases (such as epilepsy, stroke, and physical disease) that can affect research results, and (6) immediate relatives with a history of headache. In addition, to minimize the effect of hormone levels on cortical excitability, MRI scans of all female subjects were performed mid-menstrual cycle with the exception of pregnancy and lactation. Patients were headache free for at least $48 \mathrm{~h}$, fasted for $4 \mathrm{~h}$, and were not allowed coffee, tea, alcohol, cocoa, and tobacco within $12 \mathrm{~h}$ before the start of the study. Such as in our previous study, all patients were assessed by the Hamilton Anxiety Scale (HAMA), the Hamilton Depression Rating Scale (HAMD), the Montreal Cognitive Assessment screening test (MOCA), the Headache Impact Test-6 (HIT-6), the Migraine Disability Assessment Questionnaire (MIDAS), and the Visual Light Sensitivity Questionnaire-8 (VLSQ-8). The latter contains eight questions to assess the presence and severity of visual light sensitivity [28]. All HC subjects were evaluated for HAMA, HAMD and MOCA scores. No significant differences occurred between the two groups in education level, age, gender, HAMA score, HAMD score and MOCA score.

\subsection{MRI data acquisition}

All subjects underwent a PCASL scan on an Ingenia 3.0T MR system (Philips Medical Systems, Netherlands) with the application of a standard eight-channel digital head coil receiver. During the MRI scans, participants wore headphones and earplugs, and lied on their backs. A moderately tight, comfortable foam cushion was used to reduce head movement. In addition, participants were required to rest peacefully and close their eyes. All subjects fasted for at least 4 hours and were prohibited to take any vasoactive drugs for one week before the scan. The resting-state perfusion imaging technique was conducted with the application of a PCASL sequence as follows: repetition time $=4000 \mathrm{~ms}$; label duration $=1650 \mathrm{~ms}$; echo time $=11 \mathrm{~ms}$; flip angle $=90^{\circ}$; post-label delay $=1600 \mathrm{~ms}$; field of view $=240$ $\mathrm{mm} \times 240 \mathrm{~mm}$; slice thickness $=4 \mathrm{~mm}$ with $10 \%$ gap; matrix $=64 \times 64 ; 20$ axial slices; total scan duration $=4 \mathrm{~min} 08 \mathrm{~s}$.

\subsection{Analysis of MRI data and calculation of normalized CBF values}

The ASL image data were analyzed by the Statistical parameter mapping software (SPM8) (https://www.fil.ion.ucl.ac.uk/spm/software/spm8/) and the ASL data processing toolbox ASLtbx (https://cfn.upenn.edu/ zewan). The detailed procedures for the calculation of CBF maps were as described in our previous study[29]. Briefly, according to the motion parameters provided by SPM, participants with a translation greater than $2 \mathrm{~mm}$ and a rotation greater than $2^{\circ}$ were excluded from the 
analysis. The CBF images were subjected to nonlinear transformation using SPM8, and were coregistered with the PET-perfusion template in Montreal Neurological Institute (MNI) space. Each CBF coregistered in this manner was spatially trimmed to an $8 \mathrm{~mm} \times 8 \mathrm{~mm} \times 8 \mathrm{~mm}$ FWHM of the Gaussian curve. The normalization of results was performed by dividing the value of cerebral blood flow per voxel by the average cerebral blood flow through the whole brain. After data processing, 15 patients and 2 healthy subjects were excluded due to movement and subsequent image distortion, resulting in the final enrolment of 40 patients and $42 \mathrm{HC}$ subjects.

\subsection{Comparison of normalized CBF between MwoA and HC}

The difference in normalized CBF (default gray matter template) between MwoA and HC was studied using a two-sample t-test. Correction was performed using the false discovery rate (FDR) with a voxel level of $p=0.001$.

\subsection{Determination of CBF connectivity}

Considering previously used research methods, clusters with significant differences in CBF between groups were selected as regions of interest to detect whether different brain regions featured any abnormal CBF connections that corresponded to changes in CBF [30]. The CBF value of each ROI was extracted from the CBF map obtained for each patient. The CBF connection between each ROI and all other voxels in the intact brain was calculated by a multiple regression model for each group, where the confounding covariates were gender, age, and education. This step allowed for the identification of voxels that were in either positive or negative correlation with the CBF of each ROI in every group. The method of FDR was utilized to correct multiple comparisons $(p=0.001)$. Each region of interest featured two sets of CBF connection maps, which were merged into a spatial mask. Subsequently, the CBF value of each voxel was paired with that of the two sets of regions of interest. The correlation of CBF values between the two groups can possibly have different slopes for any pair of voxels; the variation in CBF connectivity between the two groups is reflected in this manner. In order to show these differences in CBF connections between MwoA patients and $\mathrm{HC}$ subjects, a specific T comparison was established in the spatial mask of the CBF connectivity map of the ROI.

\subsection{Correlation analysis using the evaluation scores}

Aiming to determine the relationships between the value of CBF and the corresponding clinical parameters, a Pearson's correlation analysis was conducted to compare the earlier established CBF value of the brain area featuring pathological changes with the patients' HAMA score, HAMD score, MoCA score, VAS score, HIT-6 score, MIDAS score and VLSQ-8 score, as well as with the disease duration and monthly attack frequency. A significance level of $P=0.05$ was adopted as the threshold for correlation analysis.

\subsection{Statistical analysis}

The Kolmogorov-Smirnov test was applied to test the normality of clinical and population baseline data distribution. The two-sample $T$ test (for age) and $\chi 2$ test (for gender) were used to analyze differences 
between groups. The Mann-Whitney $U$ test was employed to analyze measurement data with non-normal distribution (i.e., education level, HAMA score, HAMD score, MoCA score, and VLSQ-8 score). For the correlation analysis, the normalized $\mathrm{CBF}$ value of each brain region showing significant differences between the groups was extracted for each subject. The relationships between CBF value and clinical parameters were determined by Pearson correlation analysis, while the age, gender and education level were adjusted. The above statistical analysis was performed using SPSS 19.0 software (version 19.0, SPSS Inc., Chicago, IL, USA). Spm12 software was used to analyze CBF and CBF connectivity between groups. The errors were corrected by the false discovery rate (FDR) method. $P=0.001$ was considered as the threshold of statistical significance.

\section{Results}

\subsection{Participants and clinical data}

Table 1 summarizes the demographic and clinical information of the study subjects. No significant differences in age, gender and education level was shown between the two groups (all $p$ values $>0.05$ ). 
Table 1

Demographic and clinical characteristics of participants included in the study

\begin{tabular}{|c|c|c|c|}
\hline & MwoA patients $(n=40)$ & Healthy controls $(n=42)$ & $P$ value \\
\hline Age(years) & $35.10 \pm 9.48$ & $41.05 \pm 9.95$ & 0.992 \\
\hline Gender(male/female) & $10: 30$ & $15: 27$ & 0.208 \\
\hline MoCA score & $26.15 \pm 1.39$ & $26.15 \pm 1.81$ & 0.817 \\
\hline HAMA score & $40.00 \pm 7.54$ & $40.20 \pm 7.78$ & 0.857 \\
\hline HAMD score & $41.71 \pm 9.63$ & $41.49 \pm 9.44$ & 0.066 \\
\hline Education(years) & $14.18 \pm 2.61$ & $13.18 \pm 2.91$ & 0.190 \\
\hline Duration(years) & $9.20 \pm 5.82$ & NA & NA \\
\hline Headache laterality, $\mathrm{n}(\%)$ & & NA & NA \\
\hline unilateral & $18(45 \%)$ & NA & NA \\
\hline bilateral & $14(35 \%)$ & NA & NA \\
\hline shift & $8(20 \%)$ & NA & NA \\
\hline Frequency $(\mathrm{d} / \mathrm{m})$ & $5.38 \pm 2.36$ & NA & NA \\
\hline VAS & $5.03 \pm 1.53$ & NA & NA \\
\hline Mild, n(\%) & $10(25 \%)$ & NA & NA \\
\hline Moderate, n(\%) & $23(57.5 \%)$ & NA & NA \\
\hline Severe, $\mathrm{n}(\%)$ & $7(42.5 \%)$ & NA & NA \\
\hline HIT-6 score & $56.70 \pm 9.54$ & NA & NA \\
\hline MIDAS score & $10.38 \pm 8.03$ & NA & NA \\
\hline VLSQ-8 score & $21.00 \pm 5.48$ & $11.80 \pm 2.85$ & $<0.001$ \\
\hline \multicolumn{4}{|c|}{$\begin{array}{l}\text { MoCA Montreal Cognitive Assessment, HAMA Hamilton anxiety scale, HAMD Hamilton depression } \\
\text { scale, HC healthy control, Visual Analogue Scale scale 0-10: mild1-3; moderate4-6; severe7- } \\
\text { 10.HIT-6 Headache Impact Test-6, MIDAS the Migraine Disability Assessment Score, VLSQ-8 Visual } \\
\text { Light Sensitivity Questionnaire-8. Measurement data are expressed in mean and standard deviation. }\end{array}$} \\
\hline
\end{tabular}

\subsection{Differences between groups in the normalized CBF values for resting state in interictal period}

The regional CBF differences between MwoA and $\mathrm{HC}$ patients are presented in Fig. 1 and Table 2. The $\mathrm{CBF}$ was raised in the right middle frontal orbital gyrus (ORBmid.R), and the right middle frontal gyrus 
(MFG.R) in MwoA patients compared with HC patients. On the contrary, the CBF in Vermis_6 of these patients was lower than that of the control group.

Table 2

Brain regions with significant group differences in normalized CBF

\begin{tabular}{|c|c|c|c|c|c|c|}
\hline & \multirow[t]{2}{*}{ Brain region } & \multicolumn{3}{|c|}{ Peak MNI coordinates } & \multirow[t]{2}{*}{ Voxel size } & \multirow[t]{2}{*}{ Peak t score } \\
\hline & & $X$ & $\mathbf{Y}$ & $\mathbf{Z}$ & & \\
\hline \multirow[t]{2}{*}{ MwoA > HC } & ORBmid.R & 28 & 36 & -20 & 155 & 6.397 \\
\hline & MFG.R & 20 & 58 & 28 & 144 & 4.547 \\
\hline $\mathrm{MwoA}<\mathrm{HC}$ & Vermis_6 & 0 & -66 & -6 & 124 & -5.085 \\
\hline
\end{tabular}

\subsection{Differences in normalized CBF connectivity between groups}

The differences in CBF connectivity are presented in Fig. 2 and Table 3. In comparison with HC patients, MwoA patients exhibited reduced CBF connectivity between ORBmid.R and the right putamen, the left superior frontal gyrus (SFG.L), the right caudate, as well as the right angular gyrus, and the CBF connectivity between ORBmid.R and the left calcarine cortex was raised. The CBF connection with MFG.R and the Vermis_ 6 as seed points did not show any significant differences between groups.

Table 3

Brain regions with significant group differences in CBF connectivity

\begin{tabular}{|lllllll|}
\hline ROI & Brain region & \multicolumn{2}{l}{ Peak MNI coordinates } & Voxel size & Peak t score \\
\cline { 2 - 5 } & & $\mathbf{X}$ & $\mathbf{Y}$ & $\mathbf{Z}$ & & \\
\hline ORBmid.R & Calcarine.L & -2 & -66 & 20 & 102 & 3.8408 \\
& Angular.R & 50 & -48 & 32 & 72 & -5.1188 \\
& Caudate.R & 20 & 12 & 16 & 17 & -3.9317 \\
& Putamen.R & 34 & -2 & -6 & 304 & -4.3331 \\
& SFG.L & -26 & 50 & 0 & 69 & -4.9305 \\
\hline
\end{tabular}

Thresholds were set at a corrected $p<0.001$ corrected by FDR criterion. CBF: cerebral blood flow; ROI: region of interest; MNI: Montreal Neurological Institute; ORBmid.R: right middle frontal orbital gyrus; SFG.L: left superior frontal gyrus. 


\subsection{Correlations between normalized CBF values and clinical scale assessment}

The correlation analysis showed that the monthly attack frequency score and the VLSQ-8 score were positively correlated with the increased CBF in ORBmid.R (Fig. 3). Meanwhile, the correlations between further clinical parameters and changes in CBF in the MFG.R and Vermis_6 of MwoA patients (including the HAMA score, HAMD score, MoCA score, disease course, VAS score, hit6 score, and MIDAS score) proved as statistically insignificant.

\section{Discussion}

This study used 2D PCASL, a type of non-enhanced sequence, to detect changes in cerebral perfusion patterns and CBF connectivity changes of MwoA in the interictal phase. As far as the authors are concerned, this study is the first of its kind to utilize ASL-MRI for establishing the CBF connectivity patterns of MwoA patients. Furthermore, among similar studies using perfusion MRI to obtain perfusion maps of migraine during the interictal phase, our case series features the largest sample size. The trigeminal nerve vascular hypothesis, which is one of the most recognized approaches to describe the pathogenic mechanisms of migraine, states that changes in the blood flow within the brain tissue may be mediated by the secretion of certain vasoactive substances, with varying effects between different brain regions [3].

In our study, increased perfusion was detected in the ORBmid.R and MFG.R, which are key areas of the prefrontal lobe. The orbital frontal cortex (OFC) is considered as a key area for sensory integration, selfcontrol and emotional expression[31, 32]. Patients showed corresponding behavioral changes after OFC injury, such as recognition defects of emotional expression[32]. Chen et al. investigated the regional brain volume changes in episodic migraine, and showed that the right orbital frontal lobe had increased volume [33]. Compared with HC, the ALFF score of the orbital cortex in migraine patients was significantly increased during the interictal period[34]. In non-menstrual phase of primary dysmenorrhea, reduced ReHo values were observed in OFC[35]. We speculated that the increased volume, ALFF value and decreased ReHo value of orbital cortex may indicate the adaptation of the central nervous system, which could enhance descending pain modulation. The basis of these adaptations is the increase in local blood flow. Conversely, these adaptations may lead to increased local blood flow affecting the metabolism of the orbital cortex. This hypothesis can also be explained by the correlation between the attack frequency score and the CBF of ORBmid.R area, as established in our research.

Magnetic resonance imaging technology has been extensively featured in research on the connection changes in patients with migraine, such as the anatomical connection of diffusion tensor imaging, the structural connection of structural MRI, and the functional connection of functional MRI. Nonetheless, to our knowledge, no research has yet investigated the CBF connections of migraine. Although both blood oxygen level dependent (BOLD) connections and CBF connections measure the functional correlation 
among brain regions, their calculation methods are different and the results also have different physiological meanings. BOLD connectivity can be obtained by measuring the time correlation between BOLD signal fluctuations in various brain regions. Meanwhile, the calculation of CBF correlation coefficient among a group of brain regions yields the CBF connectivity. Although multiple BOLD connectivity values (one value per person) can be obtained from a given dataset, only a single such value can be established of CBF connectivity. While BOLD connectivity represents the timely synchronization of neural activity between different brain regions, CBF connectivity indicates the synchronization of perfusion or metabolism between these regions. It is worth noting that the CBF connection is regulated only by regional CBF and has a clearer physiological mechanism than BOLD connections, which are affected by hemodynamic parameters, such as cerebral blood volume and cerebral oxygen metabolic rate. Animal experiments have shown that OFC selectively connects with other prefrontal lobes (dorsolateral prefrontal cortex and medial prefrontal cortex) and the sensory cortex, including smell, taste, somatosensory, auditory and visual processing centers, and the amygdala[36]. In this study, we detected CBF disconnections between ORBmid.R and the regions of right putamen, SFG.L, right caudate, right angular, as well as left calcarine cortex during MwoA interictal periods.

The putamen and caudate are important areas of the basal ganglia. Previous studies have found changes in basal ganglia volume, functional connectivity (FC), and abnormal iron deposition in migraine patients[37, 38]. Our study showed that putamen and caudate CBF connections were abnormal, and it supported the role of basal ganglia in migraine patients.

The angular gyrus, an essential member of the default mode network (DMN), is considered as the connecting hub of global information integration[39]. Studies have shown that the functional connection of the angular gyrus is abnormal in migraine with visual aura[40]. In visual snow patients, there is an enhanced resting state functional connection between the prefrontal lobe and the angular gyrus[41]. Consistently with previous studies, we also identified decreased CBF disconnections between the ORBmid.R and the angular gyrus. These studies showed that the angular gyrus significantly contributes to visual information processing and the sensory cortical network.

Much of the primary visual cortex (BA 17) is hidden from view within the banks of the calcarine sulcus[42]. Significantly increased functional connectivity between the right thalamus and the left calcarine cortex was reported in the study of Wei et al., which also showed a positive correlation between the neural activation of the left calcarine cortex and the visual analogue scale scores[43]. The FC between the cerebellum and the left calcarine cortex also increases in migraine patients[44]. In line with previous findings, we also identified CBF disconnections between ORBmid. $R$ and the left calcarine cotex. Photophobia is a common accompanying symptom in migraine patients, while visual abnormality is the most common aura symptom in migraine patients with aura[5]. The abnormal CBF connectivity of the calcarine cotex and angular gyrus can help us better understand the pathophysiological basis of photophobia in migraine patients. Accordingly, the disconnection between ORBmid.R and these areas may be related to functional defects in optical signal processing in MwoA patients. The CBF of ORBmid.R 
was positively correlated with the VLSQ-8 score, which may indicate interactions between optical signal processing and the perception regulatory network.

The vermis is located within the spinocerebellum and receives somatic sensory input from the head and proximal body parts via ascending spinal pathways. Cerebellar vermis atrophy in hemiplegic migraine has been previously detected by MRI[45]. Vermis atrophy has also been found in patients with familial hemiplegia migraine (FHM)[46]. Functional MRI also revealed an increase in the apparent diffusion coefficient median values. $\mathrm{N}$-acetyl aspartate (NAA) and glutamate (Glu), was were significantly reduced while myo-inositol $(\mathrm{ml})$ was significantly elevated in the vermis in patients with FHM[47]. In the present study, we identified decreased CBF in the vermis for the first time in paroxysmal migraine. However, the function of the vermis remains unclear, which necessitates further research.

This study has certain limitations. Firstly, only interictal MwoA patients were enrolled, thus we cannot speculate on whether there is a difference between MwoA and MWA patients. Secondly, we only performed a single scan for each patient, thereby the dynamic perfusion changes during different phases of a migraine attack and post-attack were not gauged. Thus, we might repeatedly scan migraine patients at several time points in future studies. Finally, only the regions with statistical differences in CBF between groups were selected as the ROI for CBF connectivity analysis, which may lead to potential loss of data. Therefore, follow-up research will consider the whole brain CBF connection analysis method.

\section{Conclusions}

In summary, this study used ASL-MRI to detect changes in the CBF of multiple cortical regions, which may be one of the bases for pathophysiological changes in MwoA. Abnormal CBF connectivity between ORBmid.R and the regions of right putamen, SFG.L, right caudate, right angular, as well as left calcarine cortex was revealed for the first time in MwoA. These areas involve information perception, information integration, and emotional, pain, and visual processing. These results may provide important clues for elucidating the pathophysiology of migraine. As a whole, changes in CBF and CBF connectivity emphasize the necessity of studying the underlying neuropathology of MwoA patients from the perspective of resting CBF and CBF connections.

\section{Abbreviations}

CBF: cerebral blood flow; MwoA: migraine without aura; HC: healthy control; ORBmid.R: right middle frontal orbital gyrus; VLSQ-8:Visual Light Sensitivity Questionnaire-8; ASL: arterial spin labeling; PCASL: Pseudo-continuous ASL; HAMA:the Hamilton Anxiety Scale; HAMD: the Hamilton Depression Rating Scale; MOCA: the Montreal Cognitive Assessment; HIT-6: the Headache Impact Test-6; MIDAS: the Migraine Disability Assessment Questionnaire; VLSQ-8:Visual Light Sensitivity Questionnaire-8; MFG.R: the right middle frontal gyrus; SFG.L: the left superior frontal gyrus; OFC: orbital frontal cortex; BOLD: blood oxygen level dependent; 


\section{Declarations}

Acknowledgements

We would like to thank all the migraine patients and healthy controls who participated in our research.

\section{Authors' contributions}

$X W$ and $X Y$ designed the study.DZ, $X H, Y C, P W, C M, Z M, C L$ and $C X$ performed the experiments. $D Z$ analysed the data and was major contributor in writing the manuscript. All authors read and approved the final manuscript.

\section{Funding}

This research was supported by Nanjing Science and Technology Planning Project (No. 202002056) and Basic Research Program of Jiangsu Province(No. BK20201118).

\section{Availability of data and materials}

All data and materials generated in this study are available upon request.

\section{Ethics approval and consent to participate}

The study was based on the recommendations and approval of the Human Research Ethics Committee of the Nanjing First Hospital which is in accordance with the 1964 Helsinki declaration. Written informed consent was obtained from individual.

\section{Consent for publication}

Not applicable.

\section{Competing interests}

The authors declare that they have no competing interests.

\section{References}

1. Group GBDNDC. Global, regional, and national burden of neurological disorders during 1990-2015: a systematic analysis for the Global Burden of Disease Study 2015. Lancet Neurol 2017; 16: 877-897.

2. Roncolato M, Fabbri L, Recchia G, Cavazzuti L, Visona G, Brignoli O and Medea G. An epidemiological study to assess migraine prevalence in a sample of Italian population presenting to their GPs. Eur Neurol 2000; 43: 102-106.

3. Hadjikhani $\mathrm{N}$ and Vincent M. Neuroimaging clues of migraine aura. J Headache Pain 2019; 20: 32. 
4. Solstrand Dahlberg L, Linnman CN, Lee D, Burstein R, Becerra L and Borsook D. Responsivity of Periaqueductal Gray Connectivity Is Related to Headache Frequency in Episodic Migraine. Front Neurol 2018; 9: 61.

5. Zhang D, Huang X, Su W, Chen Y, Wang P, Mao C, Miao Z, Liu C, Xu C, Yin X and Wu X. Altered lateral geniculate nucleus functional connectivity in migraine without aura: a resting-state functional MRI study. The Journal of Headache and Pain 2020; 21 :

6. Russo A, Tessitore A, Silvestro M, Di Nardo F, Trojsi F, Del Santo T, De Micco R, Esposito F and Tedeschi G. Advanced visual network and cerebellar hyperresponsiveness to trigeminal nociception in migraine with aura. Journal of Headache and Pain 2019; 20:

7. Michelle Welman FHS, Smit AE, Jongen JLM, Tibboel D, van der Geest JN and Holstege JC. Pain Experience is Somatotopically Organized and Overlaps with Pain Anticipation in the Human Cerebellum. Cerebellum 2018; 17: 447-460.

8. Gaist D, Hougaard A, Garde E, Reislev NL, Wiwie R, Iversen P, Madsen CG, Blaabjerg M, Nielsen HH, Kroigard T, Ostergaard K, Kyvik KO, Hjelmborg J, Madsen K, Siebner HR and Ashina M. Migraine with visual aura associated with thicker visual cortex. Brain 2018; 141: 776-785.

9. Yu ZB, Lv YB, Song LH, Liu DH, Huang XL, Hu XY, Zuo ZW, Wang Y, Yang Q, Peng J, Zhou ZH and Li HT. Functional Connectivity Differences in the Insular Sub-regions in Migraine without Aura: A Resting-State Functional Magnetic Resonance Imaging Study. Frontiers in Behavioral Neuroscience 2017; 11:

10. Huang X, Zhang D, Chen Y, Wang P, Mao C, Miao Z, Liu C, Xu C, Wu X and Yin X. Altered functional connectivity of the red nucleus and substantia nigra in migraine without aura. J Headache Pain 2019; 20: 104.

11. Shin JH, Kim YK, Kim HJ and Kim JS. Altered brain metabolism in vestibular migraine: comparison of interictal and ictal findings. Cephalalgia 2014; 34: 58-67.

12. Cadiot D, Longuet R, Bruneau B, Treguier C, Carsin-Vu A, Corouge I, Gomes C and Proisy M. Magnetic resonance imaging in children presenting migraine with aura: Association of hypoperfusion detected by arterial spin labelling and vasospasm on MR angiography findings. Cephalalgia 2018; 38: 949 958.

13. Miller $\mathrm{C}$ and Goldberg MF. Susceptibility-weighted imaging and computed tomography perfusion abnormalities in diagnosis of classic migraine. Emerg Radiol 2012; 19: 565-569.

14. Bambach S, Smith M, Morris PP, Campeau NG and Ho ML. Arterial Spin Labeling Applications in Pediatric and Adult Neurologic Disorders. J Magn Reson Imaging 2020;

15. Telischak NA, Detre JA and Zaharchuk G. Arterial spin labeling MRI: clinical applications in the brain. J Magn Reson Imaging 2015; 41: 1165-1180.

16. Wolf ME, Okazaki S, Eisele P, Rossmanith C, Gregori J, Griebe M, Gunther M, Gass A, Hennerici MG, Szabo K and Kern R. Arterial Spin Labeling Cerebral Perfusion Magnetic Resonance Imaging in Migraine Aura: An Observational Study. J Stroke Cerebrovasc Dis 2018; 27: 1262-1266. 
17. Xu G, Rowley HA, Wu G, Alsop DC, Shankaranarayanan A, Dowling M, Christian BT, Oakes TR and Johnson SC. Reliability and precision of pseudo-continuous arterial spin labeling perfusion MRI on 3.0 T and comparison with 150-water PET in elderly subjects at risk for Alzheimer's disease. NMR Biomed 2010; 23: 286-293.

18. Havsteen I, Damm Nybing J, Christensen H and Christensen AF. Arterial spin labeling: a technical overview. Acta Radiol 2018; 59: 1232-1238.

19. Hernandez-Garcia L, Lahiri A and Schollenberger J. Recent progress in ASL. Neuroimage 2019; 187 : 3-16.

20. Floery D, Vosko MR, Fellner FA, Fellner C, Ginthoer C, Gruber F, Ransmayr G, Doerfler A, Uder M and Bradley WG. Acute-onset migrainous aura mimicking acute stroke: MR perfusion imaging features. AJNR Am J Neuroradiol 2012; 33: 1546-1552.

21. Linn J, Freilinger T, Morhard D, Bruckmann $\mathrm{H}$ and Straube A. Aphasic migraineous aura with left parietal hypoperfusion: a case report. Cephalalgia 2007; 27: 850-853.

22. Oberndorfer S, Wober C, Nasel C, Asenbaum S, Lahrmann H, Fueger B and Grisold W. Familial hemiplegic migraine: follow-up findings of diffusion-weighted magnetic resonance imaging (MRI), perfusion-MRI and [99mTc] HMPAO-SPECT in a patient with prolonged hemiplegic aura. Cephalalgia 2004; 24: 533-539.

23. Jacob A, Mahavish K, Bowden A, Smith ET, Enevoldson P and White RP. Imaging abnormalities in sporadic hemiplegic migraine on conventional MRI, diffusion and perfusion MRI and MRS. Cephalalgia 2006; 26: 1004-1009.

24. Mourand I, Menjot de Champfleur N, Carra-Dalliere C, Le Bars E, Roubertie A, Bonafe A and Thouvenot E. Perfusion-weighted MR imaging in persistent hemiplegic migraine. Neuroradiology 2012; 54: 255260.

25. Corno S, Giani L, Lagana MM, Baglio F, Mariani C, Pantoni L and Lovati C. The brain effect of the migraine attack: an ASL MRI study of the cerebral perfusion during a migraine attack. Neurol Sci 2018; 39: 73-74.

26. Younis S, Christensen CE, Vestergaard MB, Lindberg U, Tolnai D, Paulson OB, Larsson HB, Hougaard $A$ and Ashina M. Glutamate levels and perfusion in pons during migraine attacks: A 3T MRI study using proton spectroscopy and arterial spin labeling. J Cereb Blood Flow Metab 2020; $271678 \times 20906902$.

27. Gil-Gouveia R, Pinto J, Figueiredo P, Vilela PF and Martins IP. An Arterial Spin Labeling MRI Perfusion Study of Migraine without Aura Attacks. Front Neurol 2017; 8: 280.

28. Verriotto JD, Gonzalez A, Aguilar MC, Parel JA, Feuer WJ, Smith AR and Lam BL. New Methods for Quantification of Visual Photosensitivity Threshold and Symptoms. Transl Vis Sci Technol 2017; 6: 18.

29. Li F, Lu L, Shang S, Chen H, Wang P, Haidari NA, Chen YC and Yin X. Cerebral Blood Flow and Its Connectivity Deficits in Mild Traumatic Brain Injury at the Acute Stage. Neural Plast 2020; 2020: 2174371. 
30. Melie-Garcia L, Sanabria-Diaz G and Sanchez-Catasus C. Studying the topological organization of the cerebral blood flow fluctuations in resting state. Neuroimage 2013; 64: 173-184.

31. Rudebeck PH and Murray EA. The orbitofrontal oracle: cortical mechanisms for the prediction and evaluation of specific behavioral outcomes. Neuron 2014; 84: 1143-1156.

32. Jonker FA, Jonker $C$, Scheltens $P$ and Scherder EJ. The role of the orbitofrontal cortex in cognition and behavior. Rev Neurosci 2015; 26: 1-11.

33. Yu S-Y, Chen X-Y, Chen Z-Y, Dong Z and Liu M-Q. Regional volume changes of the brain in migraine chronification. Neural Regeneration Research 2020; 15: 1701.

34. Wang JJ, Chen X, Sah SK, Zeng C, Li YM, Li N, Liu MQ and Du SL. Amplitude of low-frequency fluctuation (ALFF) and fractional ALFF in migraine patients: a resting-state functional MRI study. Clin Radiol 2016; 71: 558-564.

35. Jin L, Yang X, Liu P, Sun J, Chen F, Xu Z, Qin W and Tian J. Dynamic abnormalities of spontaneous brain activity in women with primary dysmenorrhea. J Pain Res 2017; 10: 699-707.

36. Cavada C, Company T, Tejedor J, Cruz-Rizzolo RJ and Reinoso-Suarez F. The anatomical connections of the macaque monkey orbitofrontal cortex. A review. Cereb Cortex 2000; 10: 220-242.

37. Rocca MA, Messina R, Colombo B, Falini A, Comi G and Filippi M. Structural brain MRI abnormalities in pediatric patients with migraine. J Neurol 2014; 261: 350-357.

38. Neeb L, Bastian K, Villringer K, Israel H, Reuter U and Fiebach JB. Structural Gray Matter Alterations in Chronic Migraine: Implications for a Progressive Disease? Headache 2017; 57: 400-416.

39. de Pasquale F, Della Penna S, Snyder AZ, Marzetti L, Pizzella V, Romani GL and Corbetta M. A cortical core for dynamic integration of functional networks in the resting human brain. Neuron 2012; 74: 753-764.

40. Lo Buono V, Bonanno L, Corallo F, Pisani LR, Lo Presti R, Grugno R, Di Lorenzo G, Bramanti P and Marino S. Functional connectivity and cognitive impairment in migraine with and without aura. $J$ Headache Pain 2017; 18: 72.

41. Aldusary N, Traber GL, Freund P, Fierz FC, Weber KP, Baeshen A, Alghamdi J, Saliju B, Pazahr S, Mazloum R, Alshehri F, Landau K, Kollias S, Piccirelli M and Michels L. Abnormal Connectivity and Brain Structure in Patients With Visual Snow. Front Hum Neurosci 2020; 14: 582031.

42. Johns $₫$ Paul. Clinical Neuroscience. Churchill Livingstone, 2014.

43. Wei HL, Zhou X, Chen YC, Yu YS, Guo X, Zhou GP, Zhou QQ, Qu LJ, Yin X, Li J and Zhang H. Impaired intrinsic functional connectivity between the thalamus and visual cortex in migraine without aura. $J$ Headache Pain 2019; 20: 116.

44. Liu H-Y, Lee P-L, Chou K-H, Lai K-L, Wang Y-F, Chen S-P, Chen W-T and Wang S-J. The cerebellum is associated with 2-year prognosis in patients with high-frequency migraine. The Journal of Headache and Pain 2020; 21:

45. Ohmura K, Suzuki Y, Saito Y, Wada T, Goto M and Seto S. Sporadic hemiplegic migraine presenting as acute encephalopathy. Brain Dev 2012; 34: 691-695. 
46. Yabe I, Kitagawa M, Suzuki Y, Fujiwara K, Wada T, Tsubuku T, Takeichi N, Sakushima K, Soma H, Tsuji S, Niino M, Saitoh S and Sasaki H. Downbeat positioning nystagmus is a common clinical feature despite variable phenotypes in an FHM1 family. J Neurol 2008; 255: 1541-1544.

47. Dichgans M, Herzog J, Freilinger T, Wilke M and Auer DP. $1 \mathrm{H}-\mathrm{MRS}$ alterations in the cerebellum of patients with familial hemiplegic migraine type 1. Neurology 2005; 64: 608-613.

\section{Figures}

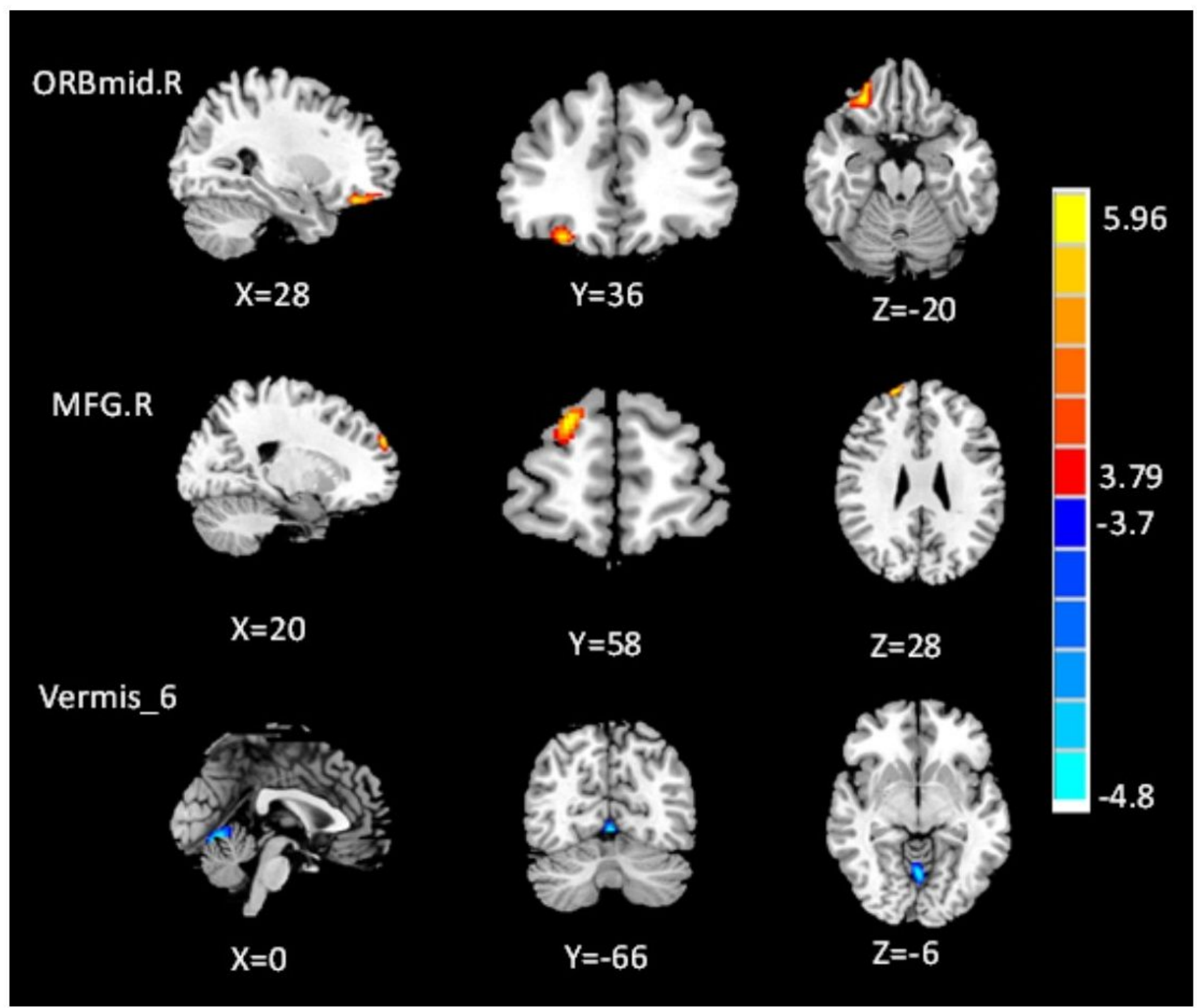

\section{Figure 1}

Voxel-based analysis indicates the brain regions with significant group differences in the normalized CBF. Compared with $\mathrm{HC}$, the MwoA patients showed increased CBF in the right middle frontal orbital gyrus (ORBmid.R), right middle frontal gyrus(MFG.R) and decreased CBF in Vermis_6. These findings 


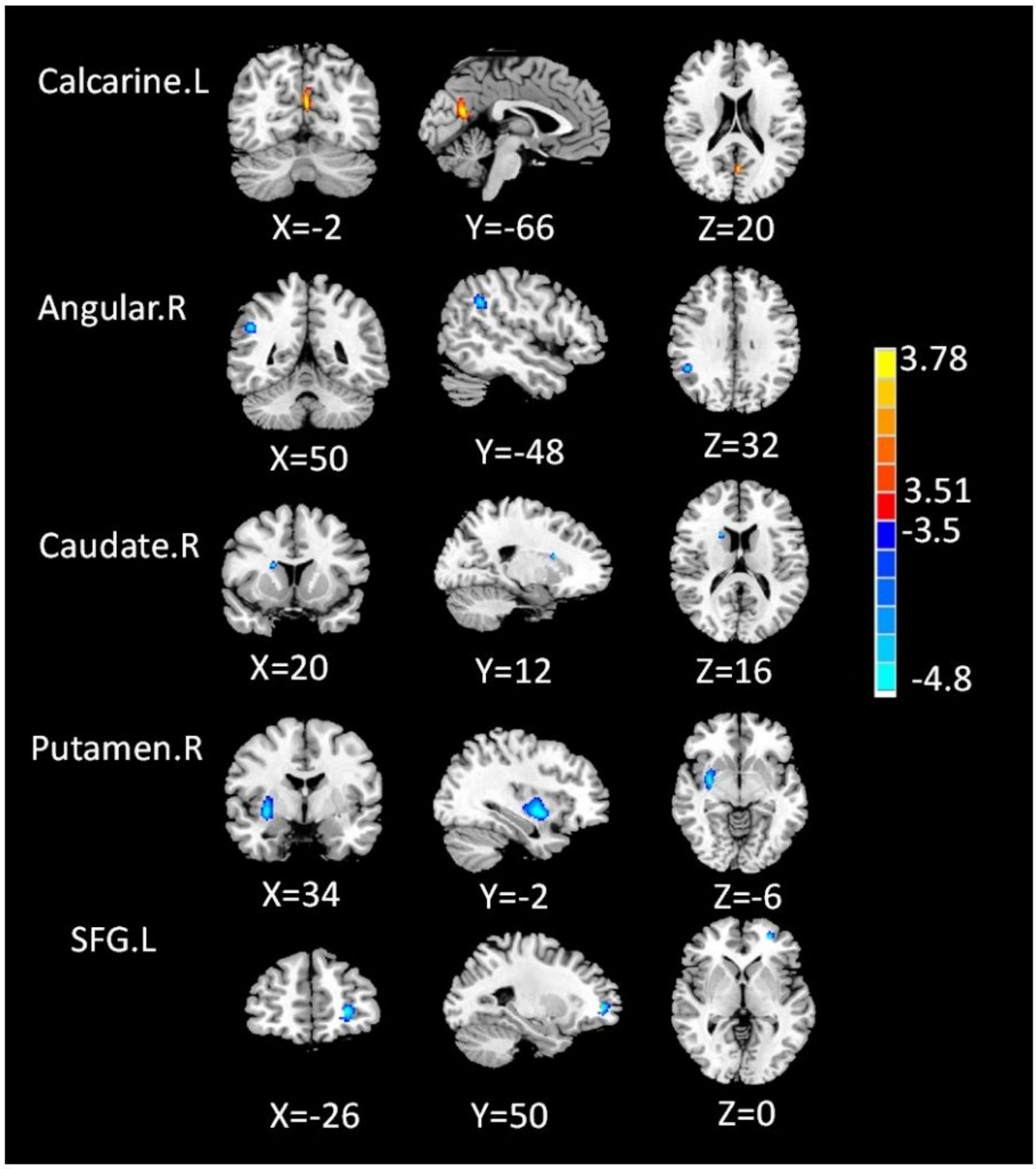

Figure 2

Compared with $\mathrm{HC}$, the MwoA patients exhibited decreased CBF connectivity between the seed ROI of the ORBmid.R and the right putamen, left superior frontal gyrus (SFG.L), right caudate as well as right angular, and increased CBF connectivity between the seed of the ORBmid.R and left calcarine cortex. 


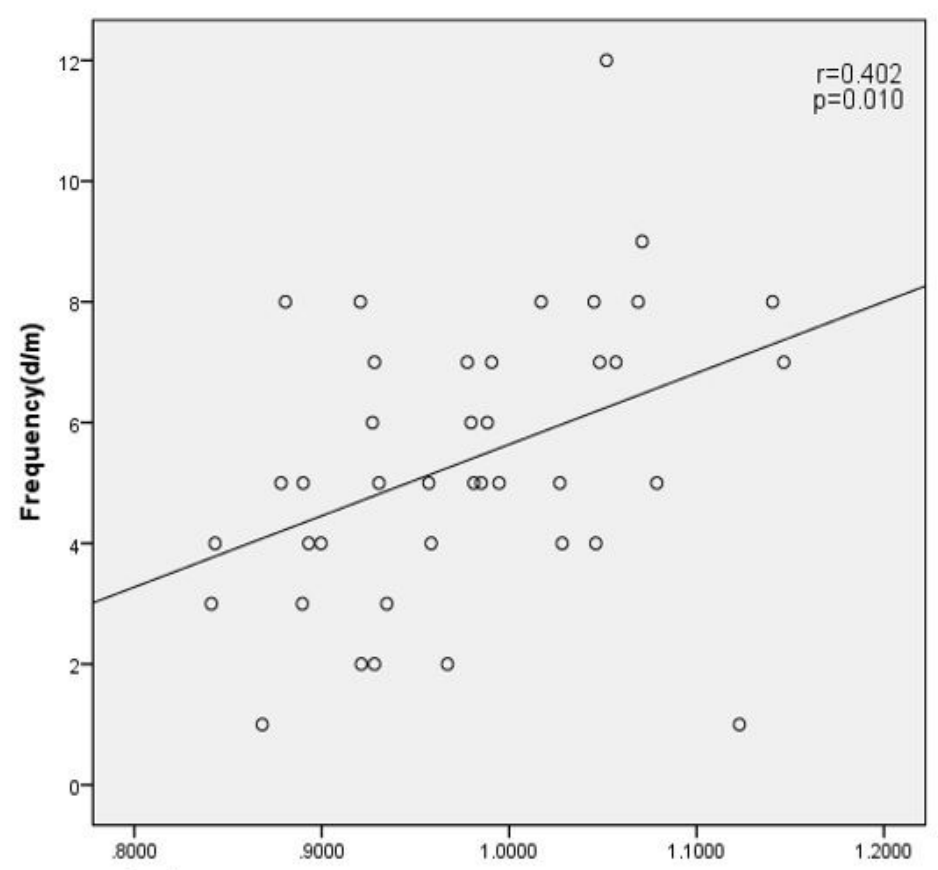

(a) Normalized CBF of right middle frontal orbital gyrus

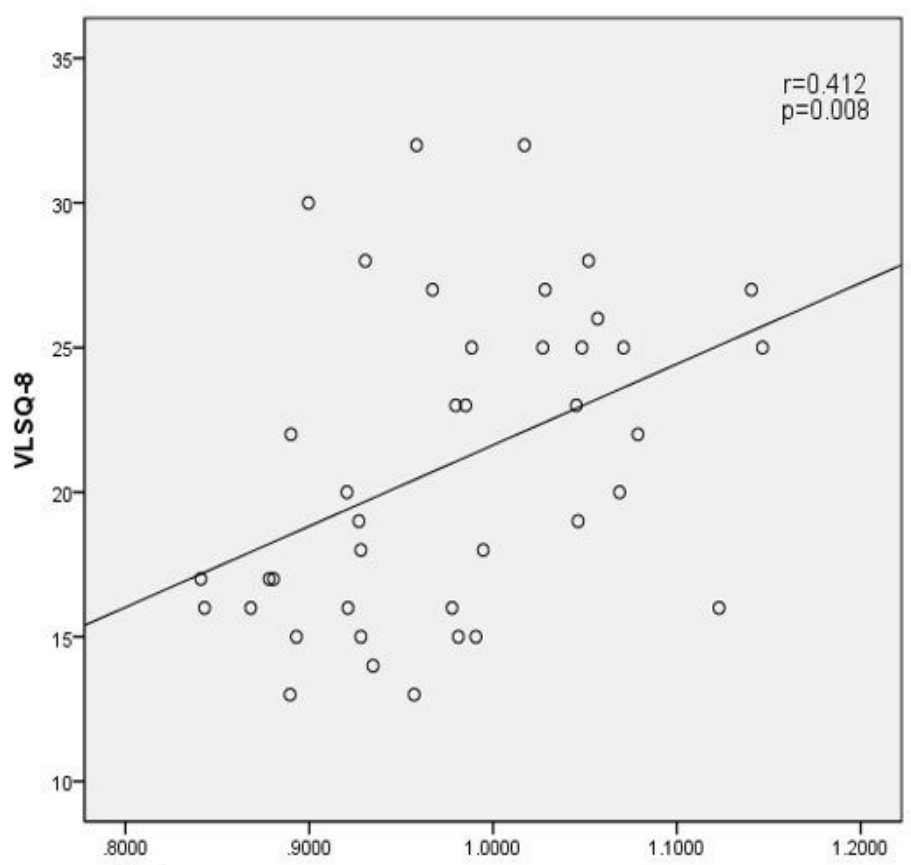

(b ) Normalized CBF of right middle frontal orbital gyrus

\section{Figure 3}

The monthly attack frequency score (a) and VLSQ-8 score (b) were positively correlated with the normalized CBF of right middle frontal orbital gyrus, respectively $(r=0.402, p=0.010 ; r=0.412, p=$ $0.008)$. 